\title{
Experimental observation of induced-charge electro-osmosis around a metal wire in a microchannel
}

\author{
Jeremy A. Levitan ${ }^{\mathrm{a}, \mathrm{b}}$, Shankar Devasenathipathy ${ }^{\mathrm{c}}$, Vincent Studer ${ }^{\mathrm{e}}$, Yuxing Ben ${ }^{\mathrm{a}, \mathrm{d}}$, \\ Todd Thorsen ${ }^{\mathrm{a}, \mathrm{b}}$, Todd M. Squires ${ }^{\mathrm{f}}$, Martin Z. Bazant ${ }^{\mathrm{a}, \mathrm{d}, *}$ \\ ${ }^{a}$ Institute for Soldier Nanotechnologies, Massachusetts Institute of Technology, Cambridge, MA 02139, USA \\ ${ }^{\mathrm{b}}$ Department of Mechanical Engineering, MIT, Cambridge, MA 02139, USA \\ ${ }^{\mathrm{c}}$ Department of Chemical Engineering, MIT, Cambridge, MA 02139, USA \\ ${ }^{\mathrm{d}}$ Department of Mathematics, MIT, Cambridge, MA 02139, USA \\ ${ }^{\text {e }}$ Laboratoire de Photonique et Nanostructures, CNRS UPR 20 Route de Nozay, Marcoussis, 91460 Paris, France \\ ${ }^{\mathrm{f}}$ Departments of Applied and Computational Mathematics and Physics, California Institute of Technology, Pasadena, CA 91125, USA
}

Available online 2 August 2005

\begin{abstract}
Induced-charge electro-osmosis (ICEO) is demonstrated around an isolated platinum wire in a polymer microchannel filled with lowconcentration $\mathrm{KCl}$, subject to a weak alternating electric field. In contrast to ac electro-osmosis at electrode arrays, which shares the same slip mechanism, ICEO has a more general frequency dependence, including steady flow in the dc limit (since the wire is not an electrode driving the field). The flow profile inside the device, measured by particle image velocimetry, confirms the predicted scaling with the square of the applied voltage, as well as the characteristic cut-off frequency. A quantitative comparison with numerical solutions of the models equations is reported for various equivalent circuit models from the literature. The standard model of linear capacitors captures the basic trends, but systematically over-predicts the velocity. A better fit to experiment can be obtained with a complex impedance, taking into account the frequency dispersion of capacitance, although the microscopic justification of this model is unclear especially in the presence of electro-osmotic flow and applied voltages well into the non-linear regime.
\end{abstract}

(C) 2005 Elsevier B.V. All rights reserved.

Keywords: Induced-charge electro-osmosis; Non-linear electrokinetics; Equivalent circuit models; Particle image velocimetry; Microfluidics

\section{Introduction}

Increased access to microfabrication technologies has spurred growing interest in the possibilities and the benefits of miniaturization in chemical analysis and biomedical screening. Microfluidics holds the promise of ultra-fast biological assays, analysis of ultra-dilute samples and characterization of a previously unrealized compounds [1-3]. In principle, these advances could be fully miniaturized, enabling new implantable medical devices and diagnostics, but there is still a need to develop robust techniques for pumping in portable microfluidic devices.

\footnotetext{
* Corresponding author. Tel.: +1 6172531713.

E-mail address: bazant@mit.edu (M.Z. Bazant).
}

Conventional pressure-driven flows are commonly used in microfluidics [6], as long as the channel dimensions are not too small $(>10 \mu \mathrm{m})$ and they have the advantage of being robust and easy to operate, regardless of the fluid composition. Nevertheless, pressure-driven flows have poor scaling with miniaturization and do not offer simple local control of flow direction and circulation. The pressure gradient needed to maintain a constant fluid velocity increases as the inverse square of the channel width and rather bulky (non-portable) pressure sources are often required.

Electrokinetics offers an alternative means to pressure gradients to drive microflows, which is gaining increased attention [4,5]. Perhaps, the best known example of electrokinetics is the electrophoresis of charged colloidal particles [7], where an applied electric field acts on diffuse double-layer charge to 
produce fluid slip. By the same mechanism, an electric field applied down a glass or polymer microchannel can drive a plug-like flow in capillary electrophoresis.

Standard electrokinetic effects are linear in the applied field, which presents some disadvantages for microfluidics, especially in miniaturization. Aside from being fairly weak, linear electro-osmotic flows suffer from the requirement of direct current and thus electrochemical reactions at electrodes to maintain a steady flow. Alternating current suppresses reactions and thus can allow larger driving voltages without bubble formation or sample contamination by reaction products, but linear flows time-average to zero in alternating fields. Another problem for miniaturization is the large power requirement of linear electro-osmosis, since the voltage is applied globally down the channel, rather than locally across the channel. As a result, to achieve typical electric fields over $100 \mathrm{~V} / \mathrm{cm}$, one must apply over $100 \mathrm{~V}$ across a $1 \mathrm{~cm}$ long microfluidic chip. It would be preferable for portable or implantable microfluidics to make use of the much smaller voltages $(1-10 \mathrm{~V})$ supplied by microbatteries by applying fields locally at the scale of the channel width.

Non-linear electrokinetic phenomena provide a promising alternative mechanism for flow control in microfluidic devices. The first non-linear electrokinetic phenomenon described in the microfluidic literature was "ac electro-osmosis" at microelectrode arrays, independently discovered by Ramos et al. [8,9] and Ajdari [10] and studied extensively ever since [11-16]. Similar flows were also observed by Nadal et al. [17] around a dielectric stripe on an electrode by Thamida and Chang [18]. Recently, Bazant and Squires [19,20] pointed out that the physical principle behind ac electro-osmosis - an electric field acting on its own induced double-layer charge at a polarizable surface - is more general, requiring neither electrodes nor ac fields and they suggested the more descriptive term, "induced-charge electro-osmosis" (ICEO) to describe the basic mechanism. They also noted that essentially the same effect had been described earlier in the Russian literature on metallic colloids by Murtsovkin and co-workers
[21,22], which speaks to the generality of the phenomenon and suggests new directions for research in microfluidics.

The fundamental effect of ICEO at an inert (non-electrode) metal surface has been predicted theoretically, but remains to be demonstrated experimentally in a microfluidic device. Here, we present experiments which reveal ICEO convection rolls around an electrically isolated platinum wire in a polymer channel driven by an ac voltage. The setup is similar to the canonical problem of a metal cylinder $[19,20]$ (or sphere [21]) in a suddenly applied uniform field, shown in Fig. 1, although the geometry is more complicated since the wire rests on one wall of the channel. We make detailed maps of the flow field inside the channel and compare with various theoretical models, solved numerically for the specific experimental geometry. Analogous experiments on metal colloids have also been reported by Gamayunov et al. [23], but the fixed and simple geometry of a microfluidic channel allows more direct, quantitative testing of the theory, as well as new technological applications.

\section{The experiment}

\subsection{Basic setup}

As a simple first experiment to illustrate ICEO flow at a non-electrode metal surface in a microfluidic device, we considered the experimental setup shown in Fig. 2. A platinum wire of circular cross-section was attached to the wall of a polymer microchannel containing low-concentration $\mathrm{KCl}$ electrolyte. An ac voltage was applied from distant ends of the channel (without any electrical connections to the wire) to produce an oscillating background electric field transverse to the wire. The observed time-averaged ICEO flow resembled that of Fig. 1(c), aside from geometrical corrections due to the channel walls, which were taken into account in the theoretical models below. The flow was visualized by tracking fluorescent tracer particles

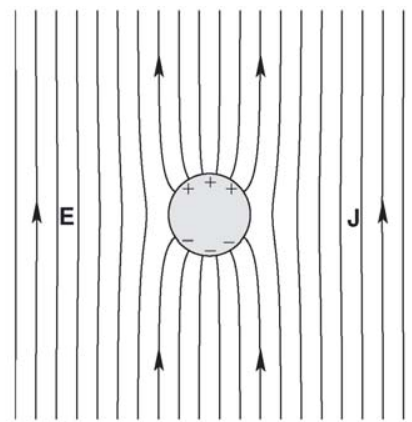

(a)

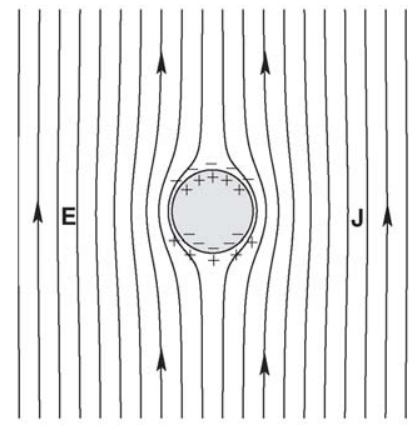

(b)

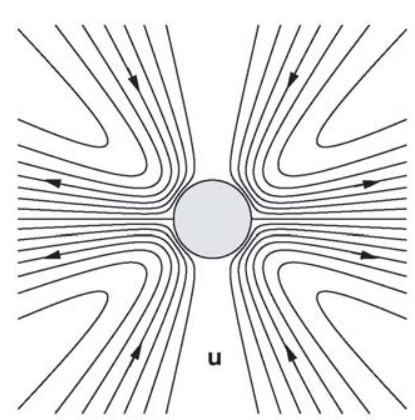

(c)

Fig. 1. Induced-charge electro-osmosis in an electrolyte around a metal wire in a suddenly applied background electric field: (a) after the near-instantaneous relaxation of electrons, the metal is an equipotential surface; (b) charging of the double layer by ionic fluxes expel the field lines, leaving a non-uniform (dipolar) charge distribution in the double layer around the metal; (c) non-linear (steady) electro-osmotic slip results as the tangential field acts on the induced double-layer charge. 


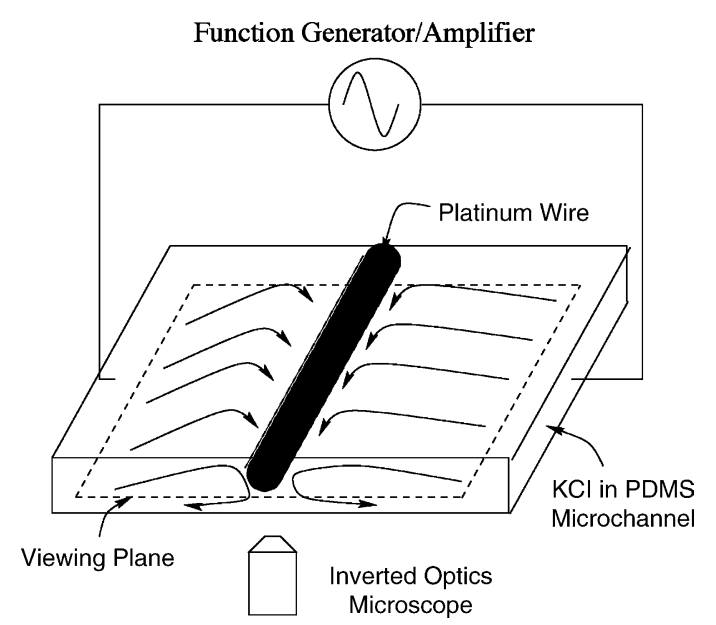

Fig. 2. Schematic diagram of the experimental setup. A $100 \mu \mathrm{m}$ diameter and $1 \mathrm{~mm}$ long platinum wire is attached to one side of a $200 \mu \mathrm{m}$ thick channel, which is $1 \mathrm{~mm}$ deep and $1 \mathrm{~cm}$ long. An ac voltage is applied across the length of channel (from left to right), transverse to the wire, by electrodes (not shown in the image) $1 \mathrm{~cm}$ apart. The time-averaged ICEO flow is visualized by imaging fluorescent tracer particles in a horizontal slice slightly below the wire (dashed plane) using an inverted optical microscope.

through an inverted optical microscope in slices transverse to the wire, allowing quantitative comparisons with the basic theory.

\subsection{Device fabrication}

Molds for the microfluidic channels were made by spincoating negative resist (Microchem SU-8 2050) onto 4 in. silicon wafers and patterned with high-resolution transparency masks. The patterned resist layer was then hard-baked. The top layer of the device was cast as a thick layer of 5:1 A:B GE Silicones (GE Silicones, RTV 615) using the master mold. The layer was cured for approximately $40 \mathrm{~min}$ at $80^{\circ} \mathrm{C}$. Ports were punched through this thick layer using 20 gauge luer stub adapters. The bottom layer of the device was 20:1 A:B GE Silicones, spun-coat at $4000 \mathrm{rpm}$ onto a \#1 glass $24 \mathrm{~mm} \times 50 \mathrm{~mm}$ cover slip and cured for $40 \mathrm{~min}$ at $80^{\circ} \mathrm{C}$. The top layer was cleaned with isopropyl alcohol and dried with nitrogen. For additional details for soft-lithographic fabrication of microchannels, the reader is referred to Refs. [24,25].

The experimental channel was $200 \mu \mathrm{m}$ thick, $1 \mathrm{~mm}$ deep and $1 \mathrm{~cm}$ long as shown in Fig. 2. The ac voltage was applied across the longest dimension of $1 \mathrm{~cm}$. The $1 \mathrm{~mm}$ long section of $100 \mu \mathrm{m}$ diameter platinum wire was cut and cleaned in acetone and isopropyl alcohol and then placed in the center of the channel on the top section. The top section was then placed on the bottom layer and the assembled layers were cured for an additional $1 \mathrm{~h}$ at $80^{\circ} \mathrm{C}$. Devices were also fabricated using Dow Corning Sylgard 184 with similar results. Various orientations of the platinum wire in the channel, for instance, laid vertically in the channel, were also achieved via this process.

\subsection{Flow generation and visualization}

To visualize the flow, small $(500 \mathrm{~nm})$ fluorescent seed particles (G500, Duke Scientific, CA) with peak excitation and emission at 468 and $508 \mathrm{~nm}$, respectively, were loaded into the microchannel at a volume loading fraction of $0.01 \%$. The neutrally buoyant polystyrene spheres were suspended in $1 \mathrm{mM} \mathrm{KCl}$ solution (prepared from granular Malinckrodt AR KCl), which has a screening length of $\lambda \approx 10 \mathrm{~nm}$. The solution was loaded into the channel with a syringe through a hollow metal fitting, which also served as the ground electrode.

The open end of the channel was capped with a $1 \mathrm{~mm}$ diameter gold wire which served as the second electrode for the oscillating voltage, applied across the channel using a function generator (33220A, Agilent, Palo Alto, CA) connected through a power amplifier (Model 50/750, Trek, Medina, NY). This setup enabled the application of between 0 and $750 \mathrm{~V}$ with a maximum frequency of $3 \mathrm{MHz}$, but the experiments reported here were restricted to $\leq 100 \mathrm{~V}$ at $\leq 500 \mathrm{~Hz}$. The typical electric field in the channel was thus in the range, $0-100 \mathrm{~V} / \mathrm{cm}$, which corresponded to an approximate background voltage drop across the $100 \mu \mathrm{m}$ wire of $0-1 \mathrm{~V}$.

An inverted microscope (Axiovert 200M, Karl Zeiss, Germany) was used to follow the tracer particles in the microchannel. The illumination source was a broad spectrum $100 \mathrm{~W}$ mercury lamp (LEJ GmbH, Germany). The fluorescent spheres were imaged through a cube filter consisting of a band-pass excitation filter (450-490 nm), dichroic mirror with a cut-off wavelength of $510 \mathrm{~nm}$ and a barrier longpass emission filter $(515 \mathrm{~nm})$. The $10 \times$ objective (numerical aperture of 0.25 ) had a measurement depth of $28 \mu \mathrm{m}$ as compared to the $200 \mu \mathrm{m}$ depth of the device [28]. The images were recorded onto a CCD camera (DFW-V500, Sony, Japan) with a 640 pixel $\times 480$ pixel array and 8-bit read-out resolution. The field of view corresponding to the CCD pixel sheet size and the magnification is $474 \mu \mathrm{m} \times 355 \mu \mathrm{m}$ in the object plane.

\subsection{Particle image velocimetry}

Particle image velocimetry (PIV) is a non-intrusive optical diagnostic technique which tracks displacements of collections of tracer particles to characterize flow fields [26]. Micro-particle image velocimetry ( $\mu$ PIV) has been implemented to map velocity fields within microfluidic devices. $\mu$ PIV utilizes fluorescent microscopy coupled with specialized cross-correlation algorithms to yield vectors with micrometer spatial resolution [27].

Fig. 3 shows a representative raw image from the experiment. The background noise, mostly attributable to fluorescent particles adsorbed to the platinum surface, is observed in this figure. Background subtraction was performed to remove the fluorescence from the stuck particles and improve signal-to-noise ratio (SNR). Images were synchronously col- 


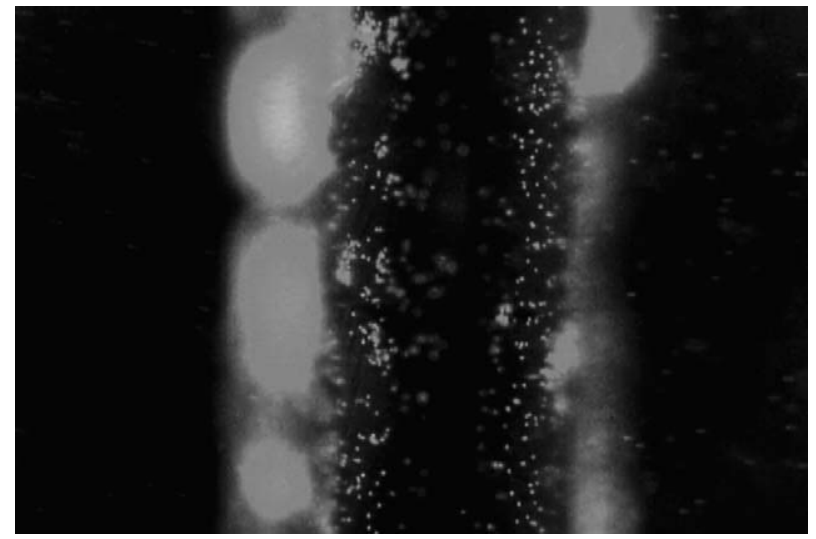

Fig. 3. An image from an optical slice, centered on a plane roughly $7.5 \mu \mathrm{m}$ away from the tip of the wire. For scale, the wire diameter is $100 \mu \mathrm{m}$. The $\mu-$ PIV analysis starts from pairs of images like these with the steady background image subtracted, including spots from clumps of trapped particles on the wire near the channel wall.

lected onto the CCD camera at 30 frames/s as movie files (AVI format) and exported as individual digital image files (TIFF format). Individual frames were analyzed with a crosscorrelation $\mu$ PIV software [29]. For the steady flows (driven by an ac field) in the present experiments, the correlations were ensemble averaged to obtain substantially higher SNR. The interrogation spots for the reported measurements were 64 pixels in the horizontal and vertical directions and with $50 \%$ overlap, a vector-to-vector spacing of $24 \mu \mathrm{m}$ was obtained in the object plane.

A sample PIV measurement of the velocity profile in an optical slice is shown in Fig. 4. The total field of view is $474 \mu \mathrm{m} \times 355 \mu \mathrm{m}$. The wire is vertically aligned at a horizontal position of 400 pixels. The width of the wire is approximately 200 pixels. Some asymmetry in the velocity profile

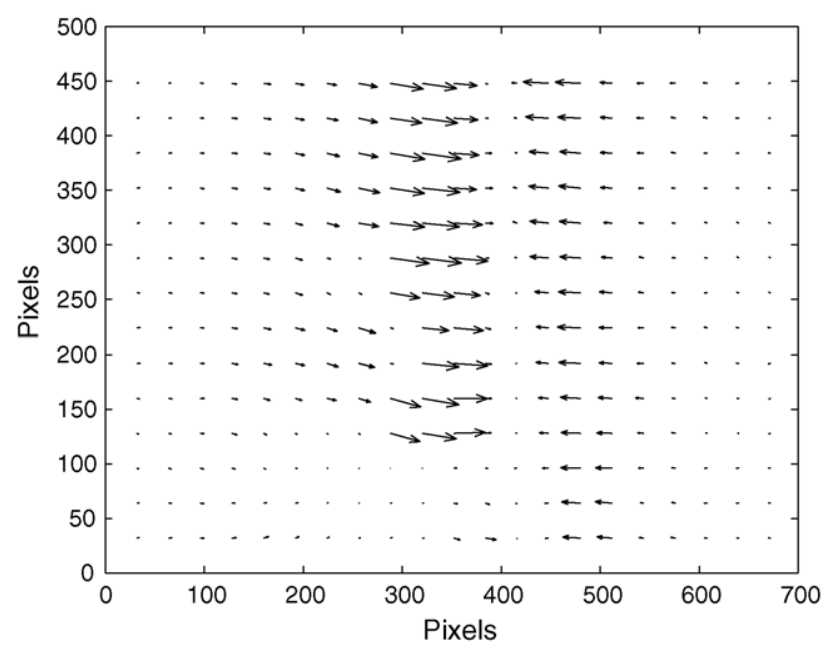

Fig. 4. A sample velocity profile obtained by particle image velocimetry, for a horizontal slice of the channel near the tip of the wire as shown in Fig. 3. The wire is roughly 200 pixels wide and centered near the 400 pixel position. is evident, which may be due to the loading of the tracer particles, from left to right, which seems to leave more trapped particles on the left, close to where the wire touches the channel wall. These trapped particles perturb the background flow and also alter the charging dynamics of the double layer near the particles. There are also clearly errors in the PIV measurement itself, which causes some cells to have much smaller velocity than neighboring cells. By ensemble averaging over many image pairs and also averaging in the symmetry direction along the wire, we are able to make fairly accurate measurements of the velocity profile transverse to the wire, with relative error less than $10 \%$.

In our experiments, the advection of the tracer particles should track the true streamlines with reasonable accuracy. Linear electrophoretic transport of the carboxylatemodified fluorescent microspheres is negligible for the applied electric field alternating at $300 \mathrm{~Hz}$. The Brownian displacement estimate for the particles over the measurement time interval is approximately $0.1 \mu \mathrm{m}$ and is reduced by ensemble averaging (error scales as the square root of the number of image pairs analyzed multiplied by the number of particles in each interrogation spot) to less than $5 \%$ of the measured displacement throughout the flow field.

The dielectric tracer particles, with slight negative surface charge, can also be subject to dielectrophoretic (DEP) forces, those resulting from gradient electric fields acting on (primarily) dielectric particles. The dielectrophoretic velocity is $\frac{\epsilon}{6 \eta} r^{2} \nabla|E|^{2}[16]$, where $\epsilon$ is the dielectric constant of the electrolyte, $\eta$ the viscosity of the fluid and $r$ is the radius of the tracking particle, while the ICEO velocity is of order $\frac{\epsilon}{\eta} E^{2} a$, where $a$ is the radius of the wire (see below). The ratio of the two velocities is thus estimated by the ratio of tracer size to wire size, squared: $U_{\mathrm{DEP}} / U_{\mathrm{ICEO}} \sim r^{2} / a^{2}$, as long as the gradient of the electric field is of order $E / a$ (which is reasonable in the absence of any sharp corners). This ratio is roughly $10^{-4}$, so, we do not expect any significant DEP on the tracer particles.

In microfluidic systems with strong electric fields and high conductivity fluids, Joule heating can be significant. As discussed by Ramos et al. [8], this heating can result in secondary flow profiles at large fields and large frequencies (typically above $10 \mathrm{kHz}$ ), although our experiments are at lower frequencies $<1 \mathrm{kHz}$. Given the highest electric field of $10^{4} \mathrm{~V} \mathrm{~m}^{-1}$ and a conductivity of $0.01 \mathrm{~S} \mathrm{~m}^{-1}$ in our setup, the power dissipation per volume $\sigma E^{2}$ can be estimated to be $10^{5} \mathrm{~W} \mathrm{~m}^{-3}$. The volume of the microchannel is $2 \times 10^{-9} \mathrm{~m}^{3}$, which implies a typical power consumption of $2 \mathrm{~mW}$, similar to the estimate in Ref. [8]. We can further calculate the temperature gradient by $\Delta T \sim \frac{\sigma V^{2}}{k}$, where $k$ is the thermal diffusion coefficient. For water, $k$ is $0.6 \mathrm{~J} \mathrm{~m}^{-1} \mathrm{~s}^{-1} \mathrm{~K}^{-1}$ and substituting a voltage of $10 \mathrm{~V}$ peak to peak leads to a temperature rise of $0.2{ }^{\circ} \mathrm{C}$. Electrothermal motion due to Joule heating typically requires larger temperature changes and higher frequencies [16], so, we conclude that this is also not an important effect in our experiments. 


\section{Theory}

\subsection{The electrochemical problem}

Given the experimental conditions, it is reasonable to test the simplest "equivalent circuit" model of ICEO, which assumes a homogeneous, neutral electrolyte with thin, charged double layers, represented by linear circuit elements. In this ubiquitous approximation [9,10,14,19-22], the electrochemical problem simplifies to that of a leaky dielectric (the bulk electrolyte) with a capacitor skin (the metal's double layer). The electrostatic potential satisfies Laplace's equation,

$\nabla^{2} \phi=0$

which is an expression of Ohm's Law, assuming no significant variations in the bulk ion concentration.

Although there may be problems with the assumption of Ohm's Law when applying large voltages across the wire (exceeding the thermal voltage, $k T / e \approx 25 \mathrm{mV}$ ), the appropriate boundary condition on the potential is even less clear [30], at least on the metal wire (the polymer channel walls are essentially non-polarizable, which yields a boundary condition of zero normal current, $\hat{n} \cdot \nabla \phi=0$ ). Here, we test three common circuit models of the wire's double layer, illustrated in parts of Fig. 5 in which: (a) Gouy's model of a linear diffuse-layer capacitance, $C_{\mathrm{D}}=\varepsilon / \lambda$; (b) Stern's model of a linear surface capacitance, $C_{\mathrm{S}}$, in series with the diffuse-layer capacitance, with a capacitance ratio, $\delta=C_{\mathrm{D}} / C_{\mathrm{S}}$; (c) the empirical model of a complex impedance, $Z(\omega) \propto(i \omega)^{-\beta}$, which describes the "frequency dispersion of capacitance" with a "constant-phase-angle", $\beta$ [31]. Theories of non-linear electrokinetics have almost invariably used model ( $a$ or $b$ ), but Green et al. [14] have recently used model (c) to improve the fit of experimental data on ac electro-osmosis (for a discussion of circuit models and their limitations, see Ref. [30] and references therein).

In the case of a capacitor model (a or b), we have the usual "RC" boundary condition, equating bulk current to doublelayer charging, on the wire's surface,

$\sigma \hat{n} \cdot \nabla \phi=C_{\mathrm{D}} \frac{\mathrm{d} \zeta}{\mathrm{d} t}$

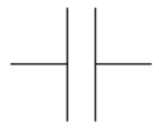

$\mathrm{C}_{\mathrm{d}}$

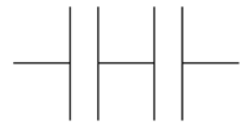

$\mathrm{C}_{\mathrm{s}}$

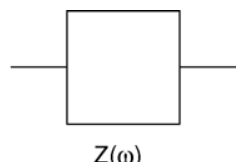

(c) (a)

(b) where $\sigma=\varepsilon D / \lambda^{2}$ is the bulk conductivity, $C_{\mathrm{D}}=\varepsilon / \lambda$ the capacitance of the diffuse part of the double layer (assumed to be constant for weak potentials), $\varepsilon$ the solvent permittivity and $\zeta$ is the local zeta potential (voltage drop across the diffuse layer), which is non-uniform in time and space. For model (b), the zeta potential is simply related to the total voltage across the double layer by:

$\zeta=\frac{\phi_{0}-\phi}{1+\delta}$

where $\phi$ is the bulk potential just outside the double layer and $\phi_{0}$ is the potential of the wire, taken to be zero by symmetry, assuming the wire has no net charge. The parameter $\delta$ interpolates smoothly between the Gouy-Chapman model with no compact layer $(\delta=0)$ and the Helmholtz model with no diffuse layer $(\delta=\infty)$. In addition to Stern's compact layer on the metal surface, the surface capacitance could also account for a thin dielectric coating [10,20], e.g. due to corrosion.

For the complex impedance model (c), we have:

$\sigma \hat{n} \cdot \nabla \phi=\frac{\phi-\phi_{0}}{Z(\omega)}$

where a sinusoidal time dependence $e^{i \omega t}$ is assumed (see below). Following Green et al. [14], we use a classical form for the impedance,

$Z(\omega)=\frac{A}{(i \omega)^{\beta}}$

which allows for a non-trivial phase angle between the applied current and the charging response for $0<\beta<1$, where $\beta=0$ corresponds to a resistor and $\beta=1$ to a capacitor. The microscopic justification for this empirical relation remains controversial, although it may be related to atomic scale heterogeneities on the metal surface [31].

\subsection{The fluid mechanical problem}

Whenever equivalent circuit models apply, the (bulk) fluid mechanics decouples from the electrochemical charging dynamics, therefore we will make this assumption regardless of its accuracy in our experiments (which probe the non-linear regime). This means that the only significant electrostatic body-force on the fluid occurs in the double layer, where it is subsumed into an effective slip velocity. Moreover, the assumption of constant bulk concentration means that fluid advection can be ignored in the electrochemical problem, regardless of the Péclet number (which may not be small in the bulk).

Due to the small Reynolds number in microfluidics, the fluid velocity, $\vec{u}$, satisfies Stokes' equations,

$\eta \nabla^{2} \vec{u}=\nabla p, \quad \nabla \cdot \vec{u}=0$

Fig. 5. Equivalent (linear) circuit models of the double layer from existing theories of non-linear electrokinetics: (a) a linear diffuse-layer capacitance, $C_{\mathrm{D}}$; (b) a linear surface capacitance, $C_{\mathrm{S}}$, in series with $C_{\mathrm{D}}$; (c) a complex impedance with the empirical frequency dependence, $Z(\omega)=A /(i \omega)^{\beta}$, where $\beta \approx 0.8$. where $\eta$ is the viscosity and $p$ is the pressure (although the unsteady term, $\partial \vec{u} / \partial t$, may be significant with ac forcing [20], it does not affect the time-averaged velocity, which is of pri- 
mary interest here). For weak applied voltages and thin double layers, the effective tangential slip, $u_{\|}$, at the wire surface is given by the Helmholtz-Smoluchowski formula,

$u_{\|}=\frac{\varepsilon \zeta}{\eta} \nabla_{\|} \phi$

where $\zeta$ is related to $\phi$ via Eq. (3), which yields a non-linear (squared) dependence on the applied voltage. In general, there may be some equilibrium zeta potential, $\zeta_{0}$, on the channel walls and the wire, but it can be safely neglected here because it can only contribute to linear electro-osmotic slip, which time-averages to zero in an ac field.

For the capacitor models ( $a$ and $b$ ), it is logically consistent to use Eq. (3) to determine the zeta potential, since it corresponds to the actual voltage drop across the diffuse layer. For the impedance model (c), however, the use of Eq. (3) is on much shakier ground and we do so, following Green et al. [14], only for lack of a better model. The fundamental problem is that, with an empirical form for the impedance of the total double layer, there is no longer a logical distinction between the diffuse and compact parts, while only the former contributes to fluid slip. Moreover, the standard slip formula (Eq. (7)) is derived from a microscopic model of transport and flow in the diffuse layer, which does not lead to a total impedance of the assumed form (Eq. (5)).

Therefore, we must view the use of Eq. (3) in model (c) as an ad hoc empirical formula for the fraction of the total double-layer voltage which leads to electro-osmotic flow. Note that Green et al. [14] make this point clear by defining a new parameter, $\Lambda$, equivalent to our parameter $(1+\delta)^{-1}$. It will turn out that this additional degree of freedom is more important in fitting the data than the specific form of the impedance in Eq. (5).

\subsection{Scaling and complex variables}

The equations are made dimensionless by scaling length to the wire radius, $a=50 \mu \mathrm{m}$, so that the channel has dimensionless height (i.e. 4). In the simulations reported below, the dimensionless electrode separation is taken to be the true separation of $200(1 \mathrm{~cm})$. Time is scaled to the characteristic "RC time" for charging the wire's double layer [30],

$\tau=\frac{a C}{\sigma(1+\delta)}=\frac{\lambda a}{D(1+\delta)}$

which is approximately $0.0005 \mathrm{~s}$ in the experiment. This corresponds to an angular frequency of $\omega_{0}=(2 \pi \tau)^{-1}=320 \mathrm{~Hz}$ for $\delta=0$. Potential is scaled to $V$, where $2 \mathrm{~V}$ is the amplitude of the applied ac voltage (with $\pm \mathrm{V}$ applied at each end, at $\pm L$ from the wire).

The fluid velocity is scaled to the typical scale for ICEO flow [21,19],

$U=\frac{\varepsilon a E^{2}}{\eta}$ which follows from Eqs. (3) and (7) with an induced doublelayer voltage of order the applied voltage across the wire, $E_{a}$, where $E=V / L$ is the typical applied electric field. For $E=100 \mathrm{~V} / \mathrm{cm}$ and $a=50 \mu \mathrm{m}$, the characteristic velocity is $U=3 \mathrm{~mm} / \mathrm{s}$, although this assumes a typical zeta potential of $E a=0.5 \mathrm{~V}$, very far into the non-linear regime $\left(E_{a} \gg k T / e\right)$ where the simple theory breaks down. Note that velocity can be scaled to $U$ independently from $a / \tau$ since the electrochemical and fluid problems decouple. The ratio of these two velocities, the diffuse-layer Péclet number, $U \tau / a=U \lambda / D(1+\delta)=0.03$, is quite small. Finally, pressure is scaled to $\eta U / a$.

As in a recent study of ac electro-osmosis by González et al. [32], we assume sinusoidal time dependence with (dimensionless) angular frequency, $\omega$ and solve for $\phi$ by its complex Fourier-transform $\Phi$, where $\phi=\operatorname{Re}\left(\Phi e^{i \omega t}\right)$. We also solve for the time-averaged velocity in response to the ac forcing. Abusing notation, the dimensionless problem is, therefore, to solve the following system of equations:

$\nabla^{2} \Phi=0, \quad \nabla^{2} \vec{u}=\nabla p, \quad \nabla \cdot \vec{u}=0$

subject to the boundary condition,

$u_{\|}=-\frac{1}{2(1+\delta)}|\Phi| \nabla_{\|}|\Phi|=-\frac{1}{4(1+\delta)} \nabla|\Phi|^{2}$

on the metal surface (where the factor $1 / 2$ comes from timeaveraging), $\hat{n} \cdot \nabla \Phi=0$ and $\vec{u}=0$ on the polymer channel walls and $\Phi= \pm L / a$ and $\vec{u}=0$ on the electrodes at the channel ends.

The final boundary condition determines $\Phi$ on the metal surface. According to the capacitance models (a and b), we have:

$\hat{n} \cdot \nabla \Phi=i \omega \Phi$

where $\omega$ is scaled to the characteristic RC frequency, $\omega_{\mathrm{c}}=$ $\omega_{0}$. In the case of the impedance model (c), we cast the boundary condition in a similar form,

$\hat{n} \cdot \nabla \Phi=(i \omega)^{\beta} \Phi$

by changing the frequency scale to $\omega_{\mathrm{c}}=\left(\frac{A \sigma}{a}\right)^{\beta} \cdot \omega_{0}$. We believe the experimental significance of the parameter $\omega_{\mathrm{c}}$ is more clear than that of $A$, which is used for fitting in Ref. [14].

\subsection{Numerical solution}

The model equations cannot be solved analytically for the experimental geometry, so accurate numerical solutions are obtained by the finite-element method (with the software package, FEMLAB, Comsol AB, Burlington, MA). The electrochemical and fluid problems decouple, so one solves first for the (complex) electrostatic potential to obtain the zeta potential distribution on the wire and then for the (timeaveraged) Stokes flow resulting from the ICEO slip. The number of elements is 4547 with 503 boundary elements. Mesh 
density is refined around the cylinder and increased gradually with a growth factor of 1.3. A typical solution takes a few seconds on a laptop computer.

Sample numerical solutions are shown in Fig. 6. For reference, the real part of Fourier-transformed electrostatic potential, $\operatorname{Re}(\Phi)$, is shown in (a) for the moment the voltage is initially applied, after relaxation of electrons on the metal wire, but before any ionic relaxation in the electrolyte. This is also the limit of high frequency, $\omega \gg 1$ (i.e. dimensional frequencies $\gg \tau_{\mathrm{c}}^{-1}$ ). In the opposite limit, $\omega \ll 1$, since the double-layer is completely charged at all times, $\operatorname{Re}(\nabla \Phi)$ in (b) is expelled from the wire and $\operatorname{Im}(\Phi)$ is zero. The timeaveraged flow in (c) shows two closed circulation rolls driven by ICEO slip on the wire, which draws in fluid along the field axis and ejects it into the middle of the channel. In this regime,

(a)

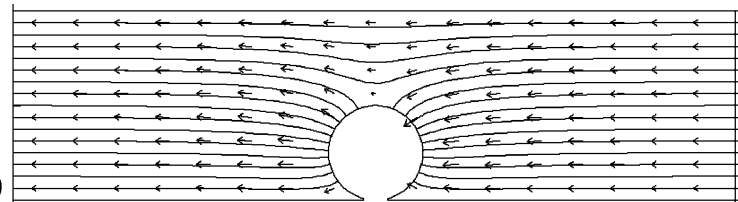

(b)

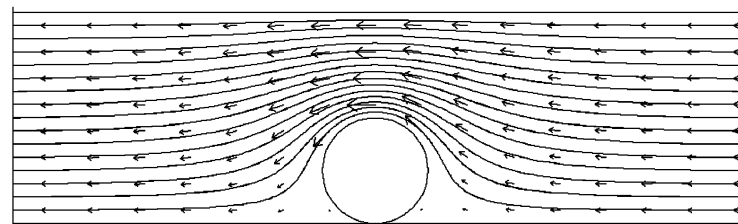

(c)

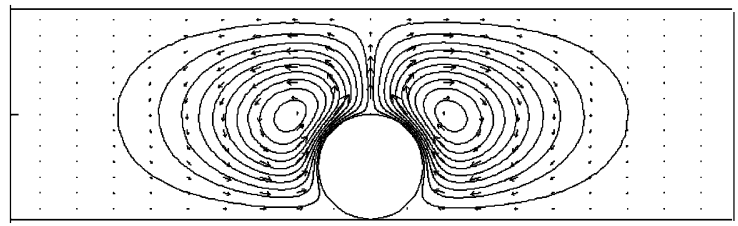

(d)

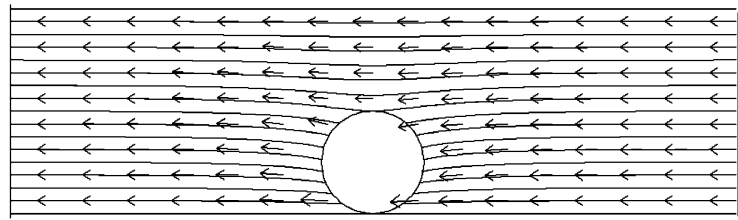

(e)

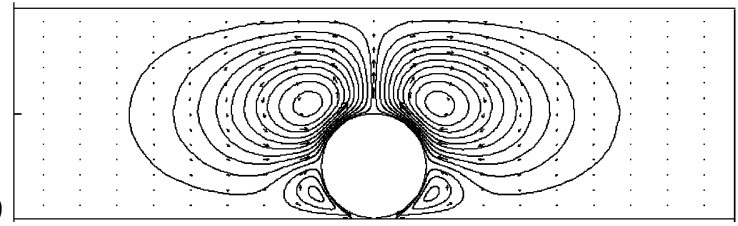

Fig. 6. Numerical simulations of induced-charge electro-osmosis in the experimental geometry. Field lines from the real part of $\nabla(\Phi)$ are shown at high frequency $(\omega=\infty)$ in (a) and at low frequency $(\omega=0.0001)$ in (b). Streamlines of the time-averaged ICEO flow in (c) for the latter case show two large vortices which draw in fluid toward the wire and expel it into the channel. At an intermediate frequency, $\omega=1, \operatorname{Re}(\nabla(\Phi))$ in (d) and flow in (e) have different profiles due to incomplete double-layer charging, which produces small, counter-rotating vortices near the wall. The arrows in $(\mathrm{a}, \mathrm{b}$ and d) indicate the direction of $\operatorname{Re}(\nabla \Phi)$ (which oscillates) and those in (c and e) indicate the (steady) time-averaged flow direction. the wire acts like a patterned metal surface on the wall generating half of the quadrupolar ICEO flow for an isolated wire in Fig. 1.

The spatial structure of the flow depends on the ac frequency. At intermediate frequencies, $\omega \approx 1, \operatorname{Re}(\nabla \Phi)$ in $(\mathrm{d})$ shows remnants of normal component due to incomplete charging. Rather than causing the field to wrap smoothly around the wire as in (b), the field $\operatorname{Re}(\nabla \Phi)$ in (d) exhibits regions close to the wall where the tangential component changes direction, pointing toward the wall. This changes the structure of the flow considerably as shown in (e). The time-averaged fluid velocity shows a new stagnation point on each side of the wire, which separates the incoming flow into one large vortex in the bulk as before and another smaller vortex against the wall, similar to the quadrupolar flow profile in Fig. 1.

\section{Results}

We begin by studying the spatial profile of the velocity from $\mu$-PIV near the tip of the wire at a driving frequency of $300 \mathrm{~Hz}$. The raw data is shown in Fig. 7 for applied voltages ranging from 35 to $100 \mathrm{~V}$. At higher voltages bubbles form at the electrodes at low frequency and at smaller voltages velocities are too small to measure accurately with our $\mu$ PIV setup.

The inverted optics microscope records images of fluorescent tracer particles in an optical slice roughly $20 \mu \mathrm{m}$ thick, set by the relatively long focal depth. To record the data, the optical slice is adjusted so as to appear just below the tip of the wire, with a clear stagnation point in the flow at the center. As shown in the sample images in Fig. 3, the optical slice also cuts partly through the top of the wire, since a thin central strip of the wire surface appears blurred. As such, we estimate the center plane of the slice to be $h=7.5 \mu \mathrm{m}$. Finally, to compare with the theory, we also adjust the horizontal position of the PIV data so that the velocity extrapolates to zero at $x=0$, consistent with the visual identification of the axis of the wire and the $10 \mu \mathrm{m}$ size of the PIV averaging cells.

Before attempting a quantitative comparison, we show in Fig. 7(b) that the data collapses well when scaled to the typical ICEO velocity, $U$ (Eq. (9)). This demonstrates that the overall velocity scales like the square of the applied voltage, as expected. The flow profiles at the smallest voltages, $V=35$ and $40 \mathrm{~V}$, show some deviation from the collapse, but this may be due to larger relative error in the $\mu$-PIV measurement. Indeed, for $V<35 \mathrm{~V}$, it is difficult to obtain a consistent value of the velocity, since many cells do not record a well defined tracer-particle displacement between successive images.

We first compare to the simplest model (a) of only a linear diffuse-layer capacitor $(\beta=1, \delta=0)$, which contains no empirical fitting parameter. The theoretical velocity profile in Fig. 7(b) clearly has the same shape as the experimental data, but its magnitude is too large by roughly a factor of three. 

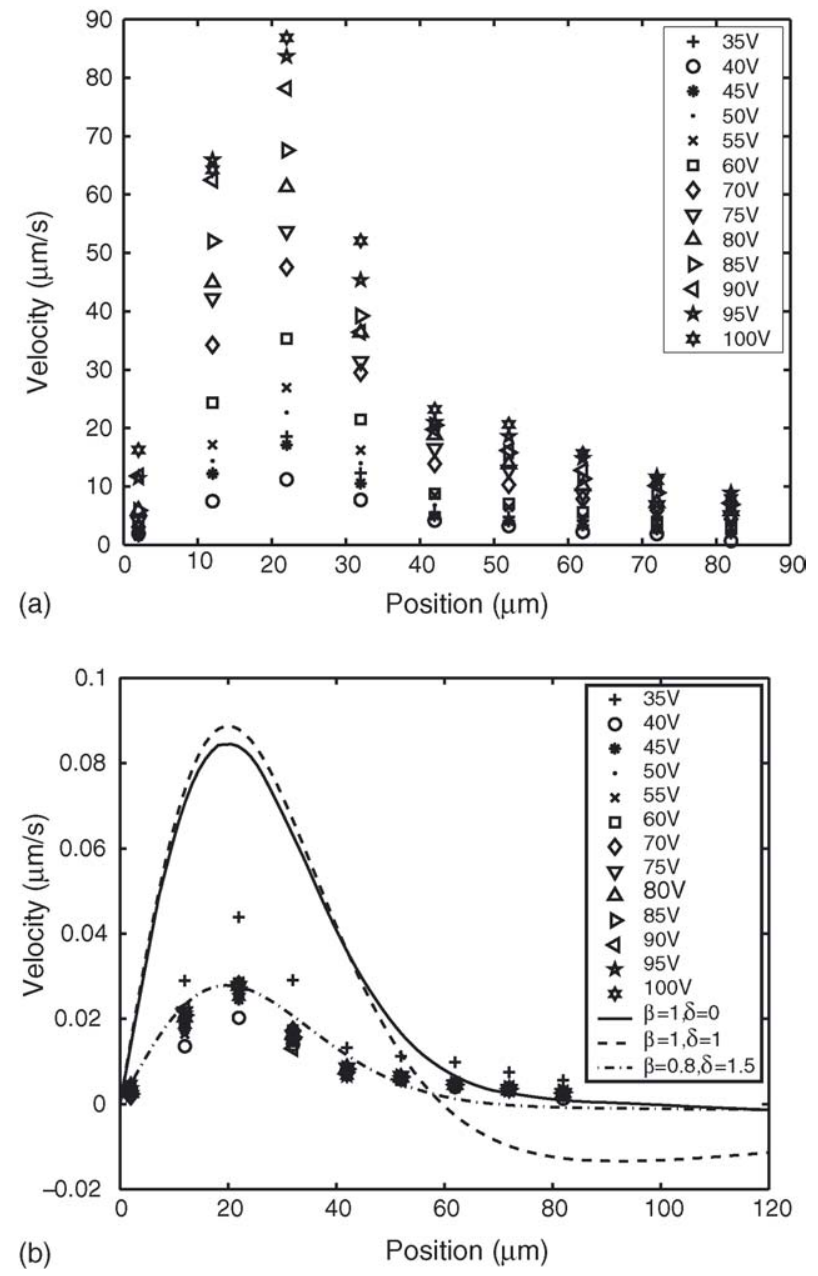

Fig. 7. ICEO velocity versus position at different voltages across $1.0 \mathrm{~cm}$ and $300 \mathrm{~Hz}$ driving signal (solid circles). Lines show simulation results for the velocity profile at $h=7.5 \mu \mathrm{m}$ above the wire surface with different $\beta$ and $\delta$ values: (a) raw data and (b) velocities are scaled by $U_{\text {ICEO }}$ in Eq. (9). In (b), $\beta$ and $\delta$ are labeled figure, $\omega_{\mathrm{c}}=320,640$ and $320 \mathrm{~Hz}$, respectively, for the solid, dash and dash-dot lines.

In the case of model (b) with $\delta=1$, which also allows for a surface capacitance, the fit is not very different for this data. In the dc limit, the theoretical velocity is reduced by a factor $(1+\delta)^{-1}$, but this effect is roughly canceled here by an increase in the characteristic frequency by a factor $(1+\delta)$, which pushes the measurement farther out of the decay regime (see below). We conclude that the data cannot be described quantitatively by the linear capacitor model (at all frequencies), although the shape of the flow profile is captured quite well. Similar conclusions have also been drawn from experiments on ac electro-osmosis at electrode arrays $[9,14,12,13]$.

To obtain a better fit, we follow Green et al. [14] and consider model (c) with a constant-phase-angle impedance. Electrochemical impedance spectra for solid electrodes typically yield values of $\beta$ in the range $0.7-0.9$ [31]. Measurements for $\mathrm{KCl}$ in contact with $\mathrm{Ti} / \mathrm{Au} / \mathrm{Ti}$ sandwich electrodes yield $0.75 \leq \beta \leq 0.82$, depending on the ion strength and the applied voltage [14]. For our $\mathrm{KCl} / \mathrm{Pt}$ system, we make a reasonable choice of $\beta=0.8$ based on these values (we will perform independent impedance measurements in our next experimental study of ICEO).

Once we have chosen $\beta=0.8$, we fit two parameters to the experimental data, $\delta$ and $\omega_{\mathrm{c}}$, even though this scheme has questionable physical justification. The additional degree of freedom makes a huge difference as we are able to achieve an excellent fit with $\delta=1.5$ and $\omega_{\mathrm{c}}=320 \mathrm{~Hz}$ as shown in Fig. 7(b). We have also checked that a fairly good fit can be achieved with other choices of $\beta$, including the purely capacitive case, $\beta=1$, as long as we still keep the two independent parameters, $\omega_{\mathrm{c}}$ and $(1+\delta)$, to scale frequency and velocity, respectively. We caution the reader, therefore, not to view our data as providing direct support for the constant-phase-angle impedance model. Instead, the availability of a second pa-
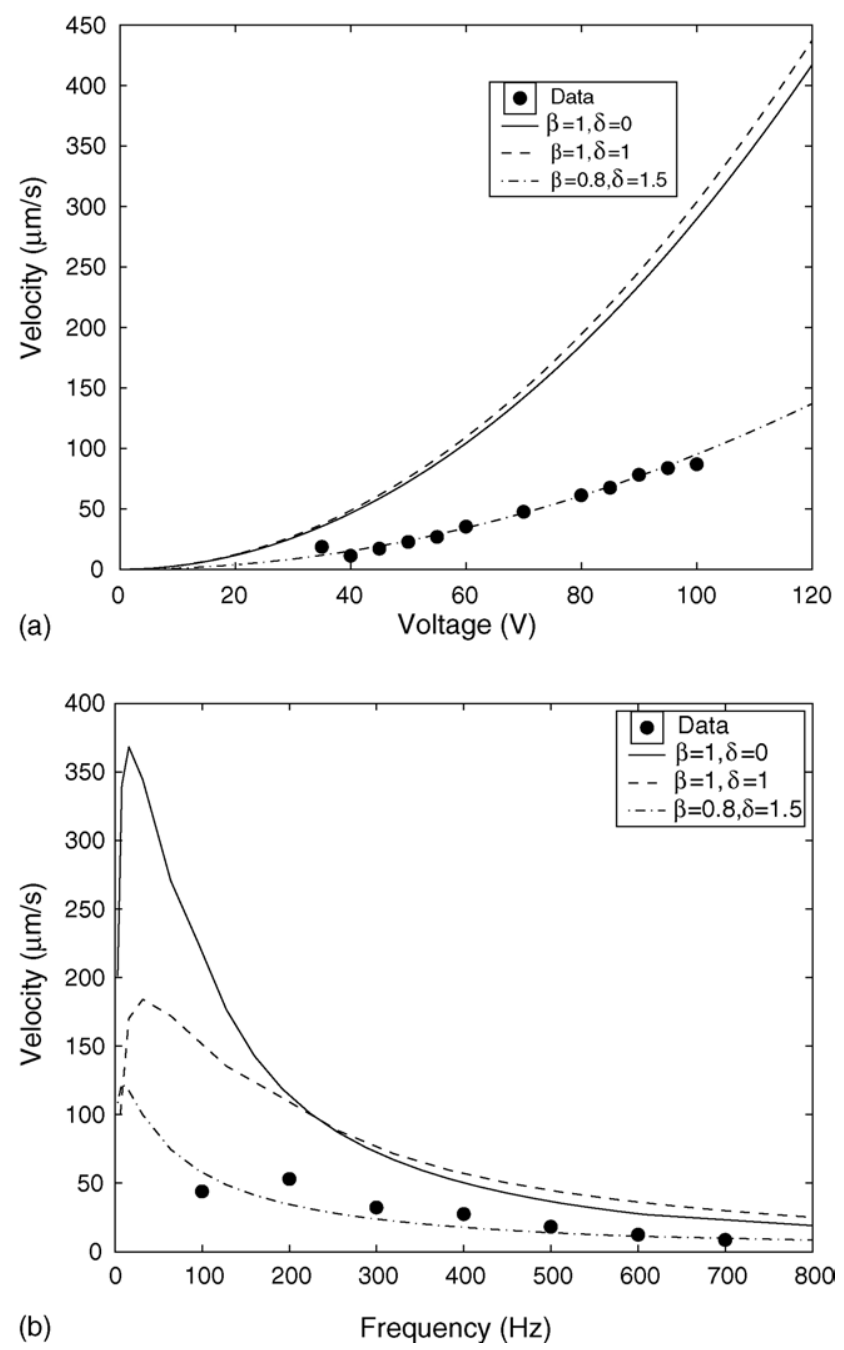

Fig. 8. Dependence of velocity on: (a) the applied voltage (across $1 \mathrm{~cm}$ ) at a fixed frequency of $300 \mathrm{~Hz}$ and (b) frequency at a fixed voltage of $50 \mathrm{~V}$. Solid circles are experimental data $22 \mu \mathrm{m}$ from the wire axis and $h=7.5 \mu \mathrm{m}$ above the wire and the lines correspond to theoretical predictions with different values of $\beta$ and $\delta$. The characteristic frequency is chosen to be $\omega_{\mathrm{c}}=320$, 640 and $320 \mathrm{~Hz}$, respectively, for the solid, dash and dash-dot lines. 
rameter to rescale the velocity seems to be the main reason for the improved fit.

The story is similar for the dependence of the velocity on voltage and frequency, sampled at the velocity maximum in the above profiles, at roughly $22 \mu \mathrm{m}$ from the wire axis. The voltage dependence in Fig. 8(a) has the correct $V^{2}$ trend in the capacitor models but the magnitude of the flow is overestimated, while the $\beta=0.8$ impedance model (due to its two free parameters) gives an excellent fit to the data. The frequency dependence in Fig. 8(b) shows roughly the expected decay at high frequencies like $\left(\omega_{\mathrm{c}} / \omega\right)^{2}$, as well as a persistence of the flow toward the dc limit of zero frequency (in the simulations, the very low-frequency regime shows flow reduction due to simple screening of the electrodes [30]). The capacitor models, extrapolated from our fits, over-estimate the velocity in the dc limit, but the discrepancy is improved by increasing $\delta$ as expected. The impedance model again gives a reasonable fit to the data, although the shape of the frequency profile seems better described by the capacitor models (if rescaled by a larger $\delta$ ).

\section{Discussion}

Overall, we find reasonable agreement between theory and experiment, sufficient to conclude that we have in fact observed ICEO. This is an interesting result on its own, since it demonstrates the same physical mechanism as ac electroosmosis around an inert (non-electrode) metal surface with a very different frequency response, including steady electroosmotic flow in the dc limit. The flow scales with the square of the applied voltage and the shape of the velocity profile and the frequency dependence are consistent with the basic theory of ICEO. Similar flows were first observed around mercury drops in ac electric fields [23], but the theory was never tested quantitatively, beyond confirming the scaling of the flow with the field squared. Microfluidics and $\mu$-PIV allows the novel possibility of controlled measurements of the complete flow field, as experimental conditions are varied.

Some difficulties arise in making quantitative comparisons between theory and experiment, as in many previous studies of linear and non-linear electrokinetics. We find that the standard capacitor model tends to over-predict the velocity by at least a factor of two under the conditions studied here. This is clearly due in part to the large applied voltages across the wire (up to $1 \mathrm{~V}$ ) which cannot be reasonably transferred to the double layer, as assumed by the simple theory. It may also have to do with the inadequate description of double-layer charging dynamics, even in the linear regime.

We have obtained good quantitative agreement between theory and experiment using an empirical model of the double-layer impedance, $Z \propto(i \omega)^{\beta}$, based on a constantphase-angle $\beta=0.8$ as in Ref. [14]. The good fit, however, seems more easily attributed to the introduction of an ad hoc fitting parameter to rescale the velocity than to any clear physical mechanism. We also find this model unsatisfactory since it not derived from the same microscopic transport equations as the Helmholtz-Smoluchowski slip formula, although perhaps closer attention must be paid to boundary conditions. It might be more self-consistent to attribute this kind of impedance to the compact layer, while retaining classical Gouy-Chapman theory in the diffuse layer.

There is also the question of the concentration dependence of ICEO flow, which we have not addressed here. In the simple Stern model (b) described here for thin double layers, the electrolyte concentration $c$ only affects the capacitance of the diffuse layer, $C_{\mathrm{D}}=\varepsilon / \lambda \propto \sqrt{c}$, which, in turn, affects the parameter $\delta \propto \sqrt{c}$. Therefore, we expect that for small concentrations, where $\delta \ll 1$, the ICEO flow speed in Eq. (9) and characteristic charging time in Eq. (8) will be insensitive to ionic strength, while for larger concentrations, where $\delta \gg 1$, the flow speed and time scale should decay like $1 / \sqrt{c}$. We have observed this rough behavior in our experiments - very little concentration dependence for $c<1 \mathrm{mM}$ and decreasing flow speed with increasing concentration for $c>1 \mathrm{mM}$ - but a more careful experimental study of the effect of ionic strength is needed, as has recently been done for ac electroosmosis $[14,13]$.

In our next experiments, we would like to improve on the setup for more accurate flow measurements. The ideal geometry would involve a nearly two-dimensional flow field to reduce the effect of uncertainty in height above the wire in the present experiments. This can be achieved with an electroplated metal cylinder in a microchannel and a microscope objective lens with a smaller measurement depth.

As mentioned above, the driving voltage in our experiments is well into the non-linear regime, where all linear circuit models lack theoretical justification. Therefore, a variety of non-linear effects should be considered in any attempt to describe more extensive experimental measurements over a wider range of operating conditions. Non-linearity tends to reduce the magnitude of ICEO flow, which grows without bound as the voltage is increased in the linear theory. In fitting our experiments, we have corrected the over-prediction of the velocity in the simplest model by introducing an empirical impedance model, but perhaps the best fit could also be improved, with clearer physical justification, by considering non-linearity in the basic electrokinetic equations.

A simple modification would be to allow for a nonlinear differential capacitance, $C_{\mathrm{D}}(\zeta)$, e.g. as predicted by Gouy-Chapman theory [30]. In the non-linear regime $\zeta>$ $2(1+\delta) k T / e$, the capacitance goes up and, thus, the charging slows down compared to the linear model, although this mainly affects the low frequency response. Other non-linear effects, which are more difficult to quantify, include changes in permittivity and viscosity due to large local electric fields in the diffuse layer.

Even with the standard assumption of constant permittivity and viscosity, a general effect of non-linearity is to violate the circuit approximation by causing ion concentrations to vary in time and space, both in the bulk and in the double layers. One such effect is the adsorption of neutral salt in the 
diffuse layer, which causes a depletion of bulk concentration with slow, diffusive relaxation [30]. Such bulk concentration gradients would generate diffusio-osmosis [7], which generally would act against the primary electro-osmotic flow. A similar mechanism is behind Dukhin's celebrated analysis of the non-linear electrophoretic mobility of highly charged particles [33]. Once concentration gradients appear, the electrochemical and fluid problems may become coupled, since the bulk Péclet number may not be small. We have estimated the double-layer Péclet number to be 0.03 , so the bulk Péclet number should be roughly $0.03 a / \lambda=150$, which is quite large. Clearly, at some point, bulk advection-diffusion will have to be addressed in non-linear electrokinetics.

A related effect is surface conduction of ions through the diffuse layer [33]. This effect can be neglected for thin double layers, as long as the induced zeta potential is not too large [20]. The relevant dimensionless parameter is the BikermanDukhin number, defined as the ratio $\sigma_{\mathrm{s}} / \sigma L$, where $\sigma_{\mathrm{s}}(\zeta)$ is the surface conductivity. This number can also be understood as controlling the relative number of ions in the diffuse layer compared to the bulk, which plays a crucial role even in onedimensional electrochemical dynamics without surface conduction and a somewhat different dimensionless number is needed in time-dependent problems [30]. The effect of the Dukhin number has been calculated for the non-linear electrophoresis of highly charged particles in weak ac fields [22], but in our experiments it is the induced charge on an initially uncharged metal surface which is large. In that case, one must consider the non-linear dynamics of diffuse charge, which is generally ignored in electrokinetic theory.

The generic effect of surface conduction is to "shortcircuit" the polarization of the double layer on a metal surface, thus reducing the induced zeta potential and the ICEO flow. In our experiments, it may be that some of the flow reduction which we have attributed to surface capacitance $(\delta=0.5-1.0)$ may instead be due to surface conduction and diffusio-osmosis. Indeed, the local Dukhin number, inferred from our simple model of the double-layer polarization, can be as large as $10^{3}$ in our experiments. Sorting out this matter will require additional experiments and more sophisticated mathematical modeling.

Another non-linear effect is the breakdown of ideal polarizability, perhaps first discussed by Murtsovkin in the context of ac electrokinetics [34]. Faradaic reactions at the metal surface can become important as the induced zeta potential is increased, eventually stealing current from the capacitive charging process. This is yet another means of "shortcircuiting" which can reduce the magnitude of ICEO flow. Some recent experiments have even observed flow reversal in ac electro-osmosis $[13,35]$, which could have something to do with Faradaic processes at the electrodes (e.g. modeled as in Ref. [36]). In our case of an isolated metal surface, we did not observe any flow reversal, although a more systematic study of the high-voltage regime could reveal some surprises.

We close by noting that, even in the simple linear regime, our simulation of the experimental geometry in Fig. 6 demon- strates an interesting effect: The spatial structure of ICEO flow in an asymmetric geometry can be controlled simply by changing the ac frequency. In contrast, for ac electrokinetics (with thin double layers) in more symmetric geometries, such as colloidal spheres [21-23] or linear electrode arrays $[8,9,11]$, changing the ac frequency primarily affects the magnitude of the velocity, but not the spatial structure of the flow, which remains unchanged for a perfect sphere or infinite electrode array (for finite electrode arrays, the stagnation points may shift, but the roll structure is fairly insensitive to ac frequency). In constrast, changing the ac frequency can significantly alter the topology of ICEO flow in an asymmetric geometry by introducing new stagnation points and closed streamlines. The ability to control the flow profile by simply varying the frequency, without changing the locations or potentials of the electrodes, might be useful in microfluidics. For example, particles might be trapped for detection or analysis in a convection roll at one frequency and then swept away to another channel at another frequency. The remarkable nonlinear sensitivity of ICEO flows to voltage, frequency and geometry merits further experimental and theoretical investigation.

\section{Acknowledgments}

This research was supported by the U.S. Army through the Institute for Soldier Nanotechnologies, under Contract DAAD-19-02-0002 with the U.S. Army Research Office (JAL, YB, TT, MZB). Some additional funding also came from the MIT-France Program (JAL, VS) as well as the NSF Mathematical Sciences Postdoctoral Fellowship and Lee A. Dubridge Prize Postdoctoral Fellowship (TMS).

\section{References}

[1] H.A. Stone, S. Kim, Microfluidics: basic issues, applications, and challenges, AICHE J. 47 (2001) 1250-1254.

[2] D.R. Reyes, D. Iossifidis, P.A. Auroux, A. Manz, Micro total analysis systems. Part 1: introduction, theory, and technology, Anal. Chem. 74 (12) (2002) 2623-2636.

[3] J. Voldman, M.L. Gray, M.A. Schmidt, Microfabrication in biology and medicine, Annu. Rev. Biomed. Eng. 1 (1999) 401-425.

[4] H.A. Stone, A.D. Stroock, A. Ajdari, Engineering flows in small devices: Microfluidics towards lab-on-a-chip, Annu. Rev. Fluid Mech. 36 (2004) 381-411.

[5] T.M. Squires, S. Quake, Microfluidics: fluid physics at the nanoliter scale. Rev. Mod. Phys. 77 (3) (2005).

[6] D.J. Laser, J.G. Santiago, A review of micropumps, J. Micromech. Microeng. 14 (2004) R35-R64.

[7] J.L. Anderson, Colloidal transport by interfacial forces, Ann. Rev. Fluid Mech. 21 (1989) 61-99.

[8] A. Ramos, H. Morgan, N.G. Green, A. Castellanos, AC electrokinetics: a review of forces in microelectrode structures, J. Phys. D Appl. Phys. 31 (18) (1998) 2338-2353.

[9] A. Ramos, H. Morgan, N.G. Green, A. Castellanos, AC electric-field induced fluid flow in microelectrodes, J. Colloid Interface Sci. 217 (2) (1999) 420-422. 
[10] A. Ajdari, Pumping liquids using asymmetric electrode arrays, Phys. Rev. E 61 (1) (2000) R45-R48.

[11] A.B. Brown, C.G. Smith, A.R. Rennie, 2001. Pumping of water with ac electric fields applied to asymmetric pairs of microelectrodes. Phys. Rev. E 63 (2), art. no. 016305.

[12] V. Studer, A. Pepin, Y. Chen, A. Ajdari, 2002. Fabrication of microfluidic devices for ac electrokinetic fluid pumping. Microelectron. Eng. 61-2, 915-920.

[13] V. Studer, A. Pépin, Y. Chen, A. Ajdari, An integrated ac electrokinetic pump in a microfluidic loop for fast tunable flow control, Analyst 129 (2004) 944-949.

[14] N.G. Green, A. Ramos, A. González, H. Morgan, A. Castellanos, Fluid flow by nonuniform ac electric fields in electrolytes on microelectrodes. Part III: observation of streamlines and numerical simulation. Phys. Rev. E 66, 026305.

[15] A. Ramos, A. Gonzales, A. Castellanos, N.G. Green, H. Morgan, Pumping of liquids with ac voltages applied to asymmetric pairs of electrodes. Phys. Rev. E 67 (2003) 056302.

[16] H. Morgan, N.G. Green, AC electrokinetics: colloids and nanoparticles, Research Studies Press Ltd., 2003.

[17] F. Nadal, F. Argoul, P. Kestener, B. Pouligny, C. Ybert, A. Ajdari, Electrically induced flows in the vicinity of a dielectric stripe on a conducting plane, Eur. Phys. J. E 9 (4) (2002) 387-399.

[18] S.K. Thamida, H.C. Chang, Nonlinear electrokinetic injection and entrainment due to polarization at nearly insulated wedges, Phys. Fluids 14 (2002) 4315-4328.

[19] M.Z. Bazant, T.M. Squires, Induced-charge electrokinetic phenomena: theory and microfluidic applications, Phys. Rev. Lett. 92 (2004) 066101.

[20] T.M. Squires, M.Z. Bazant, Induced-charge electroosmosis, J. Fluid Mech. 509 (2004) 217-252.

[21] N.I. Gamayunov, V.A. Murtsovkin, A.S. Dukhin, Pair interaction of particles in electric field. Part 1: features of hydrodynamic interaction of polarized particles, Colloid J. USSR 48 (2) (1986) 197203.

[22] V.A. Murtsovkin, Nonlinear flows near polarized disperse particles, Colloid J. 58 (3) (1996) 341-349.
[23] N.I. Gamayunov, G.I. Mantrov, V.A. Murtsovkin, Study of flows induced in the vicinity of conducting particles by an external electric field, Colloid J. USSR 54 (1) (1992) 20-23.

[24] M.A. Unger, H.-P. Chou, T. Thorsen, A. Scherer, S.R. Quake, Monolithic microfabricated valves and pumps by multilayer soft lithography, Science 288 (2000) 113-116

[25] G.M. Whitesides, A. Stroock, Flexible methods for microfluidics, Phys. Today 54 (6) (2001) 42-48.

[26] R.J. Adrian, Bibliography of particle image velocimetry using imaging methods: 1917-1995, TAM Report, University of Illinois, UrbanaChampaign, 1996.

[27] J.G. Santiago, S.T. Wereley, C.D. Meinhart, D.J. Beebe, R.J. Adrian, A particle image velocimetry system for microfluidics, Exp. Fluids 25 (4) (1998) 316-319.

[28] C.D. Meinhart, S.T. Wereley, M.H.B. Gray, Volume illumination for two-dimensional particle image velocimetry, Meas. Sci. Technol. 11 (6) (2000) 809-814.

[29] S. Devasenathipathy, J.G. Santiago, S.T. Wereley, C.D. Meinhart, K. Takehara, Particle imaging techniques for microfabricated fluidic systems, Exp. Fluids 34 (4) (2003) 504-514.

[30] M.Z. Bazant, K. Thornton, A. Ajdari, Diffuse-charge dynamics in electrochemical systems, Phys. Rev. E 70 (2004) 021506.

[31] Z. Kerner, T. Pajkossy, On the origin of capacitance dispersion of rough electrodes, Electrochim. Acta 46 (2000) 207.

[32] A. Gonzalez, A. Ramos, N.G. Green, A. Castellanos, H. Morgan, Fluid flow induced by non-uniform ac electric fields in electrolytes on microelectrodes. Part II: a linear double-layer analysis, Phys. Rev. E 61 (4) (2000) 4019-4028.

[33] S.S. Dukhin, Nonequilibrium electric surface phenomena, Adv. Colloid Interface Sci. 44 (1993) 1-134.

[34] V.A. Murtsovkin, Criterion for ideal polarizability of conducting particles, Colloid J. 53 (1991) 947-948.

[35] D. Lastochkin, R. Zhou, P. Wang, Y. Ben, H.-C. Chang, Electro-kinetic micropump and micromixer design based on ac Faradaic polarization, J. Appl. Phys. 96 (2004) 1.

[36] M.Z. Bazant, K.T. Chu,B.J. Bayly, Current-voltage relations for electrochemical thin films, SIAM J. Appl. Math. 65 (2005) 1463-1484. 